\title{
Structured Scenario-Based Design Method for Experience Vision
}

\author{
Yoshihiro Ueda ${ }^{1}$, Kentaro Go ${ }^{2}$, Katsumi Takahashi ${ }^{3}$, Seiji Hayakawa ${ }^{4}$, \\ Kazuhiko Yamazaki ${ }^{5}$, and Koji Yanagida ${ }^{6}$ \\ ${ }^{1}$ Fujitsu Design Ltd., Kawasaki, 211-8588, Japan \\ y.ueda@jp.fujitsu.com \\ ${ }^{2}$ University of Yamanashi, Kofu, 400-8511, Japan \\ go@yamanashi.ac.jp \\ ${ }^{3}$ Holon Create Inc., Yokohama, 222-0033, Japan \\ takahasi@hol-on.co.jp \\ ${ }^{4}$ Ricoh Company, Ltd., Yokohama, 222-8530, Japan \\ hayakawa@rdc.ricoh.co.jp \\ ${ }^{5}$ Chiba Institute of Technology, Narashino, 275-0016, Japan \\ designkaz@gmail.com \\ ${ }^{6}$ Kurashiki University of Science and the Arts, Kurashiki, 712-8505, Japan \\ yanagida@arts.kusa.ac.jp
}

\begin{abstract}
Experience Vision is a comprehensive design method to innovative services, systems and products which reflect upon potential stakeholders' experiences and company mission and vision. It encompasses the entire humancentered design process and presents a new vision with experiential value for both the user and business sides. It then produces users' values, activities, and interactions in scenario format as part of design activities. It finally specifies requirements specifications for the innovative services, systems and products. In this paper, we introduce a Structured Scenario-Based Design Method (SSBDM) as part of Experience Vision. SSBDM employs personas and scenarios as human-centered representations for the innovative services, systems, and products. It contains three layers of scenarios: value scenario, activity scenario, and interaction scenario. Using an example of its application in a household account book, we demonstrate how the three layers of scenarios are specified and evaluated in SSBDM.
\end{abstract}

Keywords: experience vision, service design, scenario, scenario-based design.

\section{$1 \quad$ Introduction}

The social, economic environments and technological environments - centering on the information and communications technology (ICT) environments - around us today are rapidly changing. In addition, Japan has experienced the Great East Japan Earthquake and this has accelerated movements there that aim for a safe, secure and sustainable society. There are high expectations for ways to handle social issues that 
are becoming increasingly complex, such as security measures, disaster measures and responses to problems with the global environment. To deal with environmental changes and new requirements such as these, it is thought that it will be important to not think of products, systems and services from the viewpoint of technology, but to anticipate future societies and human life and realize a vision-proposal type of design development that depicts services, systems and products for humans. To that end, we have proposed a Vision Centered Design method as a new approach to design [Fig. 1].

In the Vision Centered Design method, scenarios (which are described from the user's perspective) will be introduced as a tool to describe and convey a vision of the future. Using scenarios for the design of services, systems and products has been a commonly addressed issue in the field of Human-Centered Design (HCD).

In this paper, we introduce a Structured Scenario-Based Design Method (SSBDM). It contains three layers of scenarios: value scenario, activity scenario, and interaction scenario. Using an example of its application in a household account book, we demonstrate how the three layers of scenarios are specified and evaluated in SSBDM.

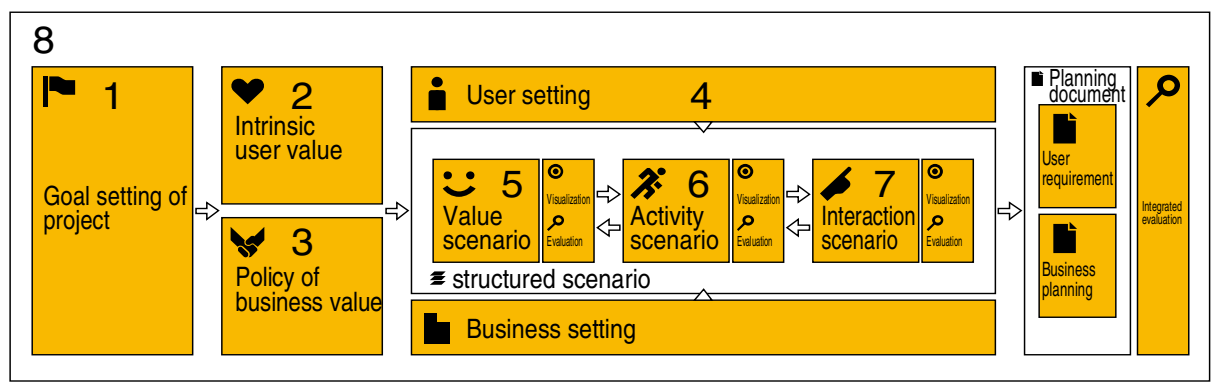

Fig. 1. Framework for Vision Centered Design Method and formats

\section{Scenario for Design}

A scenario is a story. When it is used for designing an artifact such as a service, system, or product (artifact), a scenario might contain a description of its use. For example, scenarios for designing a mobile phone might include information about who is the user, what is the mobile phone, in what situation it is used, what goal or expectation the user wishes to achieve, and how it is done. To summarize, a scenario is a description that includes actors, their background information, and assumptions about the environment, actors' goals, objectives or expectations, and sequences of actions and events. Some applications might omit one element or might express it implicitly.

\subsection{Scenario Properties for Design Development}

When conveying the general outline and features of services, systems and products, it is important to appropriately communicate the value which can be obtained by utilizing them. Explaining the practical procedures and relationships in due order will 
naturally compose a story. This is the scenario. It is used when creating ideas at an early stage of development, when explaining the idea to someone, and when keeping a written record of the idea. Thus, the scenario is first shared within the organization involved in the development. Then, when the services, systems and products are provided to the user, the scenario is utilized to explain how to use them. Promotional brochures and catalogues, for instance, explain concepts and features in a narrative form, and instruction manuals explain how to use a product step-by-step in an orderly sequence. In this way, scenarios are used in various situations that involve services, systems and products[1].

In the field of technical communication, it is conventionally said that using $5 \mathrm{~W} 1 \mathrm{H}$ (when, where, who, what, why, and how) is the most effective way to express an idea. The same applies to describing a scenario. For a product scenario, "When" and "Where" indicate the situation, "Who" indicates the user, "What" indicates the targeted services, systems and products, "Why" indicates the user's goals and expectations, and "How" indicates how to use the services, systems and products. Asking the question of "Who" leads to determining what is specifically described as a "persona," and therefore, scenarios and personas are closely related. Alternatives are comic style, or storyboard-style approaches that put photos and sketches in chronological order.

Scenarios are also effective for showing a clear vision of a concept in the phase of developing services, systems and products. The scenario indicated here will be used for subsequent development as the common viewpoint of the people involved in the development. The scenario can also be presented to the user at an early phase of development and feedback can be given on it. This shows that user evaluation is conducted at an early phase of design.

\subsection{Design Approach and Using Scenarios}

There are different ways of using scenarios in a problem-solving design approach and vision-centered design approach. In the problem-solving design approach, a scenario is used for describing the problems. If a system used by users does not meet their expectations due to an operation problem, a scenario (problem scenario) will be created to explain the problematic situation. If this situation is understood by the designer, the act of designing will be the problem-resolution tool. And if modifying the design of the system provides an immediate solution to the operation problem, a new design will be considered. In order to simply describe the new design in contrast to a problem scenario, a scenario (solution scenario) will be created to explain a situation where the users' expectations were met.

In the design action, the approach of using a scenario as a description of an intermediate product is called "scenario-based design"[2]. In some cases, scenarios are used in fragments of the design process but they can also be used as the main product.

\section{Structured Scenario-Based Design Method}

In the vision-theme design approach, however, a scenario is used to describe a vision. That is, a scenario is created to explain the future goal resulting from the 
development. In this case, the scenario will function as a development goal. Unlike the problem-solving design approach, the act of designing will not always begin based on a scenario which describes the problematic situation, and the action can begin according to the designer's free, original idea and technology seeds. As a result of the design, visualization is required to share and assess the effect on users.

\subsection{Scenario Structure}

With the vision-centered design method, the scenario will be classified according to the hierarchy and then structured. Classifying the function of a services, systems and products with the focus on its components will make it easier to understand. It can be classified into three hierarchies here: (1) value, (2) activity, and (3) interaction. This is because there are three hierarchies to actualize the overall function: (1) one that shapes value, (2) one that shapes the action to realize value, and (3) one that shapes the operation to realize action [Fig. 2]. Moreover, these hierarchies are not always structured in a top-down form - from value, to activity, and then to interaction but are also repeatedly structured and re-structured in a top-down and bottom-up form, which leads to the actualization of the eventual overall function. Based on this viewpoint, each functional hierarchy of services, systems and products is clarified by writing the scenario according to the features. This is called a "structured scenario."

(1) Value scenario - Deals with value

(2) Activity scenario - Deals with activity

(3) Interaction scenario - Deals with operation

Fig. 2. Functional hierarchy of scenario

A structured scenario is a scenario classified into one of three types which correspond to the above hierarchy: (1) value scenario, (2) activity scenario, and (3) interaction scenario. Based on these scenarios, user requirements specifications, and project proposals including business plans can be obtained.

The value scenario corresponds to the description given for value hierarchy. This scenario describes an intrinsic value for users and business value as a provider. Here, a business goal and user's expectations are pictured. In this hierarchy, rather than minute technical requirements, categories with a relatively high level of abstraction will be determined; this can be the developmental goal. In this sense, the focused evaluation viewpoint is the business viewpoint. Attraction and novelty, which reflect user satisfaction in human centered design (HCD), are also the evaluation viewpoints discussed in this hierarchy.

The activity scenario corresponds to the description given for activity hierarchy. This scenario embodies scenes where the services, systems and products are used, which are the development objects, and visualizes the user's activity. The exclusive activity flow and the user's emotions are specifically visualized as a scenario. While a business viewpoint is also described, the main objects of description are the user's concrete image and its activity. The focused evaluation viewpoint is the effectiveness in HCD. 
The interaction scenario corresponds to the description given for interaction with the hierarchy. In particular, a practical operation to make users head toward goals is pictured here, indicating the function of the services, systems and products. The focused assessing viewpoint provides efficiency in HCD.

\subsection{Features of Structured Scenario-Based Design Method}

Next, the features of each scenario in structured scenarios will be shown [Fig. 3]. With regard to the above scenarios, an image of realization from services, systems and products is described in the user requirements specifications and project proposals including business plans. The main object of description is the technology component, which is assessed from the viewpoint of feasibility. Alternatively, a structured scenario constitutionally fits the usability definition of ISO924-11. As previously stated, usability is defined as "effectiveness, efficiency, and satisfaction when a certain product is used by a designated user under a designated usage condition to achieve a designated goal."

The structured scenario clearly describes the user information in detail as a persona. The interactive scenario specifically and chronologically visualizes the users' goal and the activity process as they head towards the goal using the services, systems and products. Thus, efficiency can be evaluated here. The activity scenario describes the user experience to the abstract level which shows the overall activity flow, and effectiveness will be evaluated. The value scenario describes and assesses the users' satisfaction from the viewpoint of value provided to them. As stated above, the evaluation items in the usability definition are allocated to each hierarchy.

\begin{tabular}{|l|l|l|l|l|l|}
\hline $\begin{array}{l}\text { Type of } \\
\text { scenarios }\end{array}$ & $\begin{array}{l}\text { Business } \\
\text { goal User } \\
\text { expecta- } \\
\text { tions }\end{array}$ & $\begin{array}{l}\text { Users' } \\
\text { concrete } \\
\text { image } \\
\text { activity }\end{array}$ & $\begin{array}{l}\text { Object } \\
\text { component }\end{array}$ & Technology & $\begin{array}{l}\text { Major } \\
\text { Evaluation } \\
\text { viewpoint }\end{array}$ \\
\hline $\begin{array}{l}\text { Value } \\
\text { Scenarios } \\
\text { (User value, } \\
\text { business } \\
\text { value) }\end{array}$ & Good & Poor & Poor & Poor & $\begin{array}{l}\cdot \text { Adtraction } \\
\cdot \text { Novelty }\end{array}$ \\
\hline $\begin{array}{l}\text { Activity } \\
\text { Scenarios } \\
\text { (User } \\
\text { activity) }\end{array}$ & Average & Good & Poor & Poor & $\cdot$ HCD \\
\hline $\begin{array}{l}\text { Interaction } \\
\text { Scenarios } \\
\text { (Concrete } \\
\text { operation to } \\
\text { head towards } \\
\text { goal) }\end{array}$ & Average & Average & Good & Poor & $\cdot$ HCD \\
\hline $\begin{array}{l}\text { Project } \\
\text { proposal } \\
\text { (Implemen- } \\
\text { tation tool) }\end{array}$ & Average & Average & Average & Good & $\cdot$ Technology \\
\hline
\end{tabular}

Fig. 3. Features of each scenario in structured scenarios 


\section{Features and Example of each Scenario}

Here are the general outlines and features of the value scenario, activity scenario, and interaction scenario using a simple example. The example given here is a household account service for individual users, which will be used to differentiate between scenarios.

\subsection{Value Scenarios}

The value provided to the user is examined, generated, and described in the value scenario. As stated earlier, the necessary information is described in the value scenario focusing on the user's essential demands and the business provision policy. In particular, three items will be mainly described: 1) details of user information, 2) business information and 3) the scenario.

For details of the user, the participants involved in the services, systems and products will be listed. The user events obtained from interviews, observations, and inner comprehension will be recorded as user information. Also, the user's essential demands, which were abstracted by ranking the needs based on user information, will be described.

As for business information, the basic information on the business such as the business domain, business environment (opportunity/threat), management resources (strength/weakness), and business strategy is described. It is also effective to describe the results of SWOT (Strengths, Weaknesses, Opportunities, and Threats) analysis. In addition, there will be description of providing the policy of value based on business information, that is, providing business policy.

Lastly, the provided value will be described as a scenario. There is a particular focus on value here, and it is important not to describe the specific user image or services, systems and products. This is to keep from restricting implementation tools at the phase of examining the later services, systems and products. An example of a value scenario is shown below [Fig. 4].

\section{Value Scenario}

User Information

Beginners in handling household accounts: would like to manage the payment balance, but do not want to make too much effort.

\section{Business Information}

Has advanced image analysis technology.

Providing policy: Enable simple entry and data browsing with a high operability user interface (emphasize customer satisfaction).

\section{Scenario}

Beginners in handling household accounts accurately manage payment balance with fuss-free entry.

Fig. 4. Example of a value scenario 
When evaluating the value scenario, confirmation is required to see whether the user's essential demand and the provision policy by the business are properly reflected. The specific evaluation viewpoints are as follows:

- Whether the project goal is achieved;

- Whether the user's essential demands are satisfied;

- Whether it corresponds with the provision policy of the business; and

- Whether it is consistent with the cast.

In the value scenario, effectiveness and satisfaction from the user's perspective is recognized, and efficiency is outside the scope for this hierarchy. Similarly, business strategy attribution, business attribution, and sociality are recognized from the business perspective, but feasibility is outside the scope in this hierarchy. These outside-the-scope viewpoints are the evaluation viewpoints that depend on the implementation of products, systems and services, which are addressed in the interaction scenario.

\subsection{Activity Scenarios}

In the activity scenario, one scene is set based on the value scenario, and the overall activity flow of that scene and the user's emotion is described so that the entire image can be visualized. A concrete persona is also introduced in the activity scenario.

In particular, the details of three items - user information, scene information, and scenario - will mainly be described.

As the user information, a candidate of a persona is chosen from the cast set in the value scenario and a specific persona will be pictured. If there are multiple casts, one activity scenario will be created for each one.

The scene assumed from the value scenario is described as the scene information. A number of scenes can be assumed to realize the value depicted in the value scenario; thus one activity scenario will be created for each scene. Multiple activity scenarios will, therefore, be created from one value scenario.

Based on the above information, the activity flow of the persona in the set scene and the emotion will be described in the scenario based on the goal of the persona. Be aware that in the activity scenario, the experience of the persona is focused on and the services, systems and products are not substantiated. Therefore, the activity scenario is not dependent on implementing the object and many possibilities for implementation remain. An example of an activity scenario is shown below [Fig. 5].

A concrete image of user information is introduced in this activity scenario. The expense record is assumed as a scene. Also, Takuya's activities are described at the abstract task level. You can see his attitude from his awkwardness.

In an activity scenario, the experience of a persona in the subject scene is valued, and the activities and the user's emotion are clearly described based on the person's goal. These must reflect the content of the premising value scenario. The viewpoint, therefore, is whether these are properly reflected when evaluating the activity scenario. Specifically, the points to examine are as follows: 
- Whether the project goal is met;

- Whether (the scene written in) the value scenario is achievable; and

- Whether the persona is reflected.

In the activity scenario, the effectiveness and satisfaction from the user's perspective are recognized, and efficiency is outside the scope for this hierarchy. Similarly, the business strategy attribution, business attribution, and sociality of the business perspective are recognized, but feasibility is outside the scope in this hierarchy. These outside-the-scope viewpoints are the evaluation viewpoints that depend on the implementation of the services, systems and products, which are addressed in the interaction scenario.

\begin{tabular}{|l|}
\hline Activity Scenario \\
User Information \\
Takuya, a young second-year college student, lives in an apartment and studies \\
in the faculty of engineering at a college in the suburbs. He works hard at his part- \\
time jobs as he receives a small allowance from his parents and wants to socialize \\
with his friends. He manages his income and expenses to avoid hassles at the end \\
of the month. \\
Scene Information \\
Record expenses. \\
Scenario \\
Takuya uses this system to manage his balance. He sees the amount left in his \\
monthly budget through this system. He also records his income and expenses. He \\
wants to input the data discreetly and quickly as he feels awkward being seen re- \\
cording such small amounts of money. \\
During lunchtime, Takuya bought a boxed lunch at a convenience store near the \\
college. He paid with cash and received a bag with the boxed lunch, change, and \\
receipt. He had thrown away receipts in the past, but since he began keeping track \\
of his household account, he has been recording the amount written on the receipt. \\
\hline
\end{tabular}

Fig. 5. Example of an activity scenario

\subsection{Interaction Scenarios}

Interaction scenarios describe a scenario of concrete interactions between persona and services, systems and products written in sequential order. At this point, the functional features of the services, systems and products and the activities of the target users must be clearly defined. In particular, three items will be described mainly — details of user information, business information, and scenario.

User information is written in the same way as activity scenarios. If an activity scenario is fully complete, the description of the persona can be transcribed from the activity scenario. If there is another description about the persona, information referencing the subject persona can be indicated.

As the next step, the tasks associated with the activity scenario are detailed. Multiple tasks are assumed in order to realize the activity depicted in the activity scenario; 
thus, one interaction scenario will be created for each task. Therefore, multiple interaction scenarios can be generated from a single activity scenario.

Interaction scenarios are described based on these sets of information. Here, the relationships between the persona and services, systems and products are described in sequential order based on the task. When describing, it is especially important to express the ideas to realize the services, systems and products concretely and clearly. These realization ideas allocate functions and roles by properly factoring in the characteristic features of hardware, software, and humanware.

Interaction scenarios describe the relationships between persona and services, systems and products based on tasks in sequential order. These scenarios apparently need to contain the content of previously created activity scenarios. Therefore, an interaction scenario is evaluated from the perspective of whether these activity scenarios are properly reflected in it. Specifically, the points to examine are as follows:

- Whether the project goal is met;

- Whether (the tasks described in) the activity scenarios are realized; and

- Whether the persona is reflected.

All evaluation points are subject to evaluation in the interaction scenario. The efficiency of the user's perspective and the feasibility of the business perspective, neither of which are considered for value scenarios and activity scenarios, are assessed from the viewpoint of implementation. The following is an example of an interaction scenario [Fig. 6].

\section{Interaction Scenario}

\section{User Information}

Takuya, a young second-year college student, lives in an apartment and studies in the faculty of technology at a college located in the suburb. He works hard at his part-time jobs as he receives a small allowance from his parents and wants to socialize with his friends. He manages income and expenses to avoid hassles at the end of the month.

\section{Task Information}

Input information from receipts.

\section{Scenario}

Takuya took out his smartphone and started up an app by tapping the housekeeping book icon. A list of records was displayed in graphs and numbers by item and the current balance - 22,700 yen - was shown on the display. Takuya tapped the big "Payment" button.

The camera function was then started and a photo was taken. He took a photo by putting the camera on the receipt he had in his hands. The receipt was shown on the display and the amount paid — 480 yen — was highlighted with a thin frame line. Takuya tapped on the frame and selected "Lunch" from the menu displayed. The display changed to a record list view and he confirmed that the graph and numbers for food expenditure had increased. 
Interaction scenarios are described with concrete actions. This example illustrates an individual action to achieve the subsidiary goal - to track expense amounts which is derived from the main goal - to manage the balance of payments accurately. That is to say, it illustrates the action of inputting information from receipts. Efficiency can be evaluated because concrete actions are described in chronological order.

Project proposals containing user requirements specifications and business plans are created using the three types of scenarios above.

\section{Conclusion}

In this paper, we have described the role and characteristics of the structured scenario in the Vision Centered Design method. In addition, we have mentioned some cases and specifically given some descriptive content and shown the point of view used in evaluation for each of the three tiers in the structured scenario.

As a result of this study, we confirmed that it is possible to utilize the structured scenario method to state some ideas for vision-centered development. Moreover, we also confirmed that it is possible to elaborately expand ideas by describing the scenario in a hierarchical way. Further, we found that it is possible to describe multiple activity scenarios from one value scenario, that it is also possible to describe multiple interaction scenarios from one activity scenario, and that it is possible to generate multiple ideas and evaluate them.

We believe that a Structured Scenario-Based Design Method (SSBDM) as part of Experience Vision (Vision Centered Design method), is an effective way to efficiently produce sophisticated ideas.

\section{References}

1. Yamazaki, K., Ueda, Y., Go, K., Takahashi, K., Hayakawa, S., Yanagida, K.: Experience Vision. Maruzen Publishing Co., Ltd., Japan (2012) ISBN 978-4-621-08565-3 C 3050

2. Go, K.: What Properties Make Scenarios Useful in Design for Usability? In: Kurosu, M. (ed.) HCD 2009. LNCS, vol. 5619, pp. 193-201. Springer, Heidelberg (2009)

3. Edited by Japan Industrial Designers' Association: Product Design, Works Corporation, pp. 116-117 (2009)

4. Yanagida, K., Ueda, Y., Go, K., Takahashi, K., Hayakawa, S., Yamazaki, K.: Visionproposal Design Method. In: Kurosu, M. (ed.) HCD 2011. LNCS, vol. 6776, pp. 166-174. Springer, Heidelberg (2011)

5. Yanagida, K., Ueda, Y., Go, K., Takahashi, K., Hayakawa, S., Yamazaki, K.: Structured Scenario-based Design Method. In: Kurosu, M. (ed.) HCD 2009. LNCS, vol. 5619, pp. 374-380. Springer, Heidelberg (2009) 